\title{
Demokratische Republik und Arbeiterbewegung in der Verfassungstheorie von Wolfgang Abendroth $(1906-1985)$
}

Die Verfassungstheorie von Wolfgang Abendroth ist Teil seiner politischen Theorie. Doch nehmen die verfassungsrechtlichen Beiträge - sowohl ihrem Umfang nach als auch in der Form der wissenschaftlichen Ausführung - im Gesamtwerk Abendroths eine Sonderstellung ein. Es ist vermutlich der Teil aus seinem wissenschaftlichen Opus, der über die zeitgebundenen politischen Analysen und Positionen hinaus Bedeutung behalten wird.

Abendroths Verfassungstheorie ist bisher nur unzulänglich untersucht worden. ${ }^{1}$ Der spezifische Ansatz von Wolfgang Abendroth wurde häufig mißverstanden. Viele meinten, Abendroth »links « überholen zu müssen: Man ist gegen den "Staat" und will an die Stelle des Kampfes um Verfassungspositionen die Organisation des Klassenkampfes unter bewußtem Verzicht auf die juristische Aktion setzen. Andere haben verkannt, daß Abendroths Verfassungstheorie auf die Arbeiterbewegung bezogen ist, und entwickeln Strategien, die vor allem auf vorstaatliche Menschenrechte gestützt werden, verlieren sich (unter Ausklammerung des Zieles der gesellschaftlichen Veränderung) im moralischen Streit oder formulieren - meist folgenlos - rechtsförmige Anspruchskategorien.

Gegenüber solchen Positionen kommt es darauf an, die wesentlichen Elemente der von Abendroth entwickelten Verfassungstheorie herauszuarbeiten.

\section{Die Unterschetdung zwischen Staatsapparat und demokratischer Verfassung}

In der Abendrothschen Verfassungstheorie gibt es keine Mystifizierung des "Staates « und weder eine blinde Staatsfixierung noch eine abstrakte Staatsnegation. Wesentlich für seine Verfassungstheorie ist die Unterscheidung zwischen Staatsapparat einerseits und demokratischer Verfassung andererseits. Der "Staatsapparat " und die durch die Verfassung gesicherte "demokratische Ordnung « dürfen nicht »unkritisch gleichgesetzt « werden. ${ }^{2}$ Demokratische und soziale Teilhabe sind für Abendroth Gegenpositionen gegenüber autoritärer Exekutivmacht, gegenüber dem

\footnotetext{
I Vgl. dazu Jürgen Seifert, •Der Kampf um Verfassungsposttionen*, in: neue kritik, Nr. 35 (April 1966), S. 4-11; erweiterte Fassung in: Kampf um Verfassungspositionen, Köln, Frankfurt/M., 1974, S. 105-124, Joachım Perels, "Einleitung zu: Wolfgang Abendroth, Arbetterklasse, Staat und Verfassung a, Frankfurt/M., Köln, I975, S. 7-20; Jürgen Seifert, * Wolfgang Abendroth und die SPD *, un: Wolf-Dieter Narr, Hrsg., Auf dem Weg zum Einpartetenstaat, Opladen, 1977, S. 242-249; Volker Neumann, »Rechts- und verfassungstheoretusche Positionen der staatsrechtlichen Linken 4 , in: Der Staat, Bd. 21, H. 4, 1982, S. 55I-575; Joachim Perels, *Marxismus in der Restaurationszeit «, in: Soztalismus (Sonderheft Abendroth). November i 985 , Hamburg, S. 22-24.

2 Wolfgang Abendroth, Arbetterklasse, Staat und Verfassung, ebd., S. 165.
} 
„bürokratischen Verwaltungsstaat «. J Jede dieser Gegenpositionen ist ertrotzt. Die demokratische Verfassung und die damit verbundene demokratische Einwirkung auf Exekutivmacht und Justizapparat sind vom Bürgertum und in Deutschland weitgehend von der Arbeiterbewegung erkämpft worden. Die heute verfassungsrechtlich garantıerte demokratische Betätigung des Bürgers durchlief die drei Phasen: Verbot, Duldung, Anerkennung. Auch soziale Teilhabe, die für Abendroth immer mehr war als bloße soziale Symmetrie, muß politisch und gesellschaftlich erstritten werden. Demokratische Verfassungsrechte sind das Produkt des Kampfes gesellschaftlicher Klassen, entstehen als Kompromiß und sind in der konkreten Ausprägung das Resultat von Machtkonstellationen. ${ }^{4}$ In diesem Sinne kann die Verfassung als Waffenstillstandslinie divergierender politisch-gesellschaftlicher Kräfte bezeichnet werden.s

Abendroths Gegenüberstellung von Staatsapparat und demokratischer Ordnung ist für den politischen Kampf von größerer sozialwissenschaftlicher Trennschärfe. Der Begriff demokratischer Ordnung umfaßt sowohl den verfassungsrechtlichen Rahmen als auch die, diesen Rahmen tragenden politisch-sozialen Kräfte. Demokratische Ordnung realisiert sich im Sinn der Tradition der europäischen Arbeiterbewegung in der demokratischen Republik. ${ }^{6}$ Die demokratische Republik ist das Kampffeld, auf dem der Klassenkampf mit dem Ziel der sozialen Umwälzung ausgetragen wird. ${ }^{7}$ Dieser Kampf ist für Abendroth ein politischer Kampf. Er richter sich gegen das Kapital und zielt auf eine Veränderung der bisherigen Produktionsweise. Gegner in diesem Kampf sind auch die politischen Funktionsträger des Kapitals in konservativen Organisationen und in Teilen des Staatsapparats.

Abendroth führte den Kampf um Verfassungspositionen mit dem Ziel, die demokratische Republik (als Errungenschaft) zu verteidigen gegen jede Rückentwicklung, sei es in der Form des autorıtären Verwaltungsstaats oder eines faschistischen Regimes. Abendroth hat exakt jede Veränderung im Staatsapparat und die dadurch möglichen Gefahren für die demokratische Republik registriert. Es ging ihm nicht um die Abstraktion "Staat«, nicht um irgendwelche »Staatsableitungen«; er bezeichnete vielmehr genau die Institution und die Funktion, die er meinte, so zum Beispiel den »Polizeiapparat (soweit es um repressive Funktionen gegenüber der Arbeiterbewegung und anderen sozialen Bewegungen geht), den »Justizapparat « (soweit er von konservativen Kräften beherrscht ist) und das Militär. Er warnte vor Ausnahmebefugnissen jeder Art für die Exekutive. Solche Ausnahmebefugnisse sind für

3 S. dazu den Titeł der ersten Sammlung verfassungsrechtlicher Beiträge: Herbert Sultan, Wolfgang Abendroch, Bürokrattscher Verwaltungstaat und soztale Demokratte, Hannover, Frankfurt/M., o.J. (1955).

4 Wolfgang Abendroth, Arbetterklasse, Staat und Verfassung, a. a. O. (Anm. 1), S. 155; Wolfgang Abendroth, Antagonistische Gesellschaft und politusche Demokratze, Neuwsed, Berlin, 1967, S. 126.

s Jürgen Seifert, a.a.O. (Anm. I), S. S. xisf.; s. dazu auch S. VIII (ebd.); dort ist vom wbegrenzten Stellenwert* der *Analyse von Verfassungsbestımmungen als Kompromiß und Waffenstillstandslinea die Rede; Abendroth hat vom "Klassenwaffenstillstand * und von "Waffenstillstandslagen * gesprochen (Peter Römer, Hrsg., Der Kampf um das Grundgesetz, Frankfurt am Ma1n, 1977, S. I 88 ff.); das hat dazu geführt, daß Neumann (a.a.O., Anm. I) Abendroth und Seifert durch die Formel „Grundgesetz als Waffenstillstandsabkommen* zusammenfaßt (S. $552 \mathrm{ff}$.); Neumann verkennt, daß für Abendroth die Waffenstillstandslinie des Grundgesetzes auch inhaltlich bestımmt $15 \mathrm{t}$ : durch die demokratischen und sozialen Teilhaberechte; vgl. dazu auch Wolfgang Abendroth, , Über den Zusammenhang von Grundrechtssystem und Demokratie*, in: Joachim Perels, Hrsg., Grundrechte als Fundament der Demokratze, Frankfurt am Main, 1979, S. 249-262.

6 S. dazu Joachım Perels, $*$ Marxismus in der Restaurationszedtu, a. a. O. (Anm. I), S. 23.

7 Nachweise dazu beı Jürgen Seifert, Kampf um Verfassungspositionen, a. a. O. (Anm. I), S. I I i; vgl. ferner Annelies Laschitza, Deutsche Lınke im Kampf für eine demokratısche Republik, [Ost-]Berlin, I969. Die demokratische Republik war im Herdelberger Programm der SPD (1925) die zentrale verfassungstheoretische Kategorie. 
konservative Kräfte das Einfallstor zur Rückentwicklung der demokratischen Republik. Die demokratische Republik ist in den Augen Abendroths in Gefahr, solange Kapitalismus existiert. Sie ist bedroht, weil kapitalistische Produktionsweise und demokratische Ordnung in Krisensituationen unverembar sind: "Die Trennung des politischen und ökonomischen Kommandos (erzeugt) einen Spannungszustand ..., der im nur formaldemokratisch organisierten Staat auf die Dauer nicht aufrechterhalten werden kann. Geraten in der Demokratie in Krisenperioden die Massen in Bewegung, so werden die Inhaber nicht demokratisch legitimıerbarer ökonomischer Machtposition stets dazu neigen, die demokratische Organisation des politischen Gemeinwesens aufzuheben, um die bevorstehende Bedrohung ihrer Machtposition durch demokratische Willensbildung im Staate auszuschalten. $*^{8}$

Weil Abendroth von dieser Bedrohung der demokratischen Republik ausgeht, hängt alles von der "ständigen Bereitschaft der demokratischen Kräfte des Volkes ab «, die Demokratie zu schützen. ${ }^{9}$ Es ist verkürzt, diese theoretische Position mit dem Hinweis auf ein Faschismustrauma Abendroths abzutun.

\section{Demokratusche und soziale Teilhaberechte}

Der wesentliche Beitrag Abendroths zur Verfassungstheorie ist die Begründung demokratischer und sozialer Teilhaberechte. ${ }^{10}$ Abendroth stützt sich beim Begriff der Teilhaberechte auf die klassische Unterscheidung der amerikanischen und französischen Revolution zwischen Menschen- und Bürgerrechten. Abendroth hat, im Anschluß an Marx, die Bürgerrechte (anders als Menschenrechte im Sinn des Eigentumsrechts) als konstitutiv für die demokratische Verfassungsordnung interpretiert. Für Marx waren diese Bürgerrechte spolitische Rechte, die nur in der Gemeinschaft mit andern ausgeübt werden. Die Teilnabme am Gemernwesen, und zwar am politischen Gemeinwesen, am Staatswesen, bilden ihren Inhalt. Sie fallen unter die Kategorie der polituschen Freiheit ... ${ }^{11}$ Abendroth hat das unter Anlehnung an Hermann Heller weiter entwickelt. ${ }^{12}$ Er stellte die Frage: "Wie weit diese liberalen Grundrechte, zunächst als Ausgliederungsrechte gegenüber der Staatsgewalt formuliert und gedacht, nun, weil es sich um einen demokratischen und sozialen Rechtsstaat handelt, in Teilhaberechte umgedacht werden müssen, die

8 Wolfgang Abendroth, Antagontstzsche Gesellschaft und politzsche Demokratte, a. a. O. (Anm. 4), S. I24; Abendroth stützt sıch dabeı auf Hermann Heller, Staatslehre, Leiden, 1934, S. I38; s. dazu Wolfgang Abendroth, "Die Funktion des Politikwissenschaftlers und Staatsrechtslehrers Hermann Heller in der Weımarer Republik und in der Bundesrepublik Deutschland «, in: Chrıstoph Müller u. Ilse Staff, Hrsg., Der sozıale Rechtsstaat. Gedächtnisschrift für Hermann Heller, Baden-Baden, 1984, S. 213 ff.; vgl. auch Joachım Perels, Kapitalismus und politische Demokratse, Frankfurt am Marn, I 973.

9 Wolfgang Abendroth, Arbetterklasse, Staat und Verfassung, a. a. O. (Anm. I), S. 82; dieses Zitat steht für viele vergleschbare Äußerungen Abendroths.

is Vgl. dazu den m. E. wichtıgsten verfassungstheoretıschen Beıtrag Abendroths „Zum Begriff des demokratischen und sozialen Rechtsstaates im Grundgesetz der Bundesrepublik Deutschland a in der Festschrift für Ludwig Bergsträsser (1954), abgedruckt in: Antagontstische Gesellschaft und politrsche Demokratze, a. a. O. (Anm. 4), S. 109-1 38 u. den Diskussionsbeitrag von Abendroth auf der Tagung der Vereıngung Deutscher Staatsrechtslehrer 1953, abgedruckt in: Arbetterklasse, Staat und Verfassung, a. a. O. (Anm. I), S.64-69. Jürgen Habermas hat diesem Betrag Abendroths eine grundlegende Bedeutung zugemessen und ist der von Abendroth gestellten Frage wetter nachgegangen, Strukturwandel der Öffentlichkett, Neuwied, r962, S. 247 ff.; vgl. ferner ders., Theone und Praxts, Neuwied, 1964, S. 85 ff., Ulnch K. Preuß, Zum staatsrechtlichen Begriff des Öffentlichen, Stuttgart, 1969, S. I64ff. u. Jürgen Seifert, Grundgesetz und Restauration, 3. Aufl., Neuwied, Darmstadt, 1977, S. 54-64.

I Karl Marx, *Zur Judentrage* (1843), in: MEW, Bd. 1, S. 362.

12 Hermann Heller, Rechtsstaat oder Diktatur?, Tübingen, 1930; s. dazu Abendroth (Antagonistısche Gesellschaft und politusche Demokratze, a.a.O. (Anm.4), S. I 12. 
gleichzeitig mit dem früheren bloßen Ausgliederungscharakter dieser Grundrechte $46 I$ soweit notwendig und soweit möglich - versöhnt werden müssen. « ${ }^{13}$

Teilhaberechte umreißen für Abendroth die demokratische Teilhabe des Bürgers im Sinne der demokratischen Mitwirkung aller am Gemeinwesen und in der Gesellschaft. Für Abendroth kommt es nicht nur auf die verfassungsrechtliche Garantie eines Teilhabeanspruchs an. Demokratische Teilhabe muß in den Augen Abendroths auch chancenreich sein. Demokratische Teilhaberechte sind in diesem Sinne Rechte, durch die politische Gewalt legitimiert wird, die materiellen Voraussetzungen zu schaffen, die dem Bürger die Teilnahme an der demokratıschen Willensbildung in der Gesellschaft überhaupt erst möglich machen.

Teilhaberecht bedeutet auch soziale Teilhabe. Unter bestimmten Voraussetzungen soll es ein Anspruch auf Teilhabe an staatichen Leistungen geben. Abendroth hat das Sozialstaatspostulat ${ }^{14}$ des Grundgesetzes herausgearbeitet und dieses Postulat in Verbindung gesetzt mit dem Gleichheitssatz. Heute ist allgemein anerkannt: Wenn das Gemeinwesen aus öffentlichen Mitteln materielle Leistungen gewährt, kann es nicht der freien Entscheidung staatlicher Organe überlassen sein, den Kreis der Begünstıgten selbst zu bestimmen; vielmehr muß jeder an den durch diese Leistungen gebotenen Lebenschancen prinzipiell gleichberechtigt beteiligt sein."

Abendroths Verdienst ist es, über diese Form der sozıalen Teilhabe hinaus, Teilhaberechte interpretiert zu haben als programmatische Rechte für die politische Realisierung »einer neu zu gliedernden Gesellschaf $« .{ }^{16}$ Dabei ging es ihm um die Absicherung des politischen Anspruchs der Arbeiterbewegung, im Rahmen der demokratischen Republik die "soziale Demokratie ${ }^{17}{ }^{7}$ zu verwirklichen, nicht aber um die Einlösung juristisch formulierter Anspruchskategorien auf dem Rechtswege.

\section{Der Verfassungskampf als Teil und Wegbereiter des politzschen Kampfes}

Wolfgang Abendroths Theorie und Praxis war bezogen auf die Arbeiterbewegung. Es ging ihm nicht um das, was man $»$ Juristensozialismus $^{18}$ genannt hat. Er kämpfte um juristische Positionen, nicht weil er glaubte, auf diese Weise Sozialismus realisieren zu können, sondern darum, daß in der Bundesrepublik sozialistische Positionen vertreten werden können ${ }^{19}$ und gesellschaftsverändernde Tätigkeit möglich ist. Diese Verfassungspositionen dienen dazu, für die Arbeiterbewegung Aktionsraum zu schaffen.

Das ist teilweise von denjenigen mißverstanden worden, die die erste Restaurationsphase der Bundesrepublik mit der Verfolgung von Kommunisten und den Versuchen, sozialistisches Denken zu illegalisieren, nicht kennengelernt haben. ${ }^{20} \mathrm{Geprägt}$

13 Wolfgang Abendroth, Arbetterklasse, Staat und Verfassung, a. a. O. (Anm. 1), S. 66.

I4 Vgl. dazu Hans-Hermann Hartwich, Sozzalstaatspostulat und gesellschaftlicher status quo, Köln, Opladen, 1970 .

is Vgl. dazu BVerfGE Bd. 33 , S. 333.

16 Wolfgang Abendroth, Arbetterklasse, Staat und Verfassung, a.a. O. (Anm. 1), S.67.

17 Vgl. dazu den Schlußsatz von Wolfgang Abendroth, "Demokratie als Institution und Aufgabe (1954) in: Arbetterklasse, Staat und Verfassung, a. a. O. (Anm. I), S. 32; vgl. in diesem Zusammenhang die für Abendroth wichtige Schrift Max Adler, Politusche oder soziale Demokratie, Wien, 1926.

18 [Friedrich Engels u. Karl Kautsky], »Jurısten-Sozıalismus « (1887), in: MEW, Bd. 21, S. 491-509.

19 W. Abendroth u. a., „Diskussion über Probleme soztalistischer Rechtspolitik*, in: Hubert Rottleutner, Hrsg., Probleme marxistischer Rechtstbeone, Frankfurt am Man, 1975, S. 393.

20 So etwa Eckart Osborg u. Rainer Roth, „Wer herrscht über die Legalität? « in: Heidelberger Blätter, Diskus-Express, 1968, Heft $12 i_{3}$ (1/2), S. 7-10; vgl. auch die damalige Position von Rainer Geulen, 
durch die Erfahrungen der Weimarer Zeit und mit Blick auf verhängnisvolle Losungen von damals ("Demokratie, das ist nicht viel, Sozialismus ist das Ziel! « "Nach Hitler kommen wir! «), hat Abendroth sich dagegen gestemmt, daß der Kampf um Verfassungspositionen und der Klassenkampf auseinander dividiert und zu einem "entweder - oder « gemacht werden. ${ }^{21}$

Für Abendroth stand außer jedem Zweifel, »daß die Emanzipation der Arbeiterklasse durch die Arbeiterklasse selbst vollbracht werden muß «. ${ }^{22}$ Er war sich bewußt, daß er weder als Verfassungsjurist oder als Politikwissenschaftler noch als politisch eingreifendes Individuum stellvertretend für politisch-soziale Kräfte handeln konnte. Abendroth hat versucht, Massen zu mobilisieren und nie aufgehört, auf politische Organisationen so einzuwirken, damit diese für den Sozialismus kämpfen.

Sein Leitgedanke war, den verfassungsrechtlichen Rahmen des Grundgesetzes offenzuhaiten für gesellschaftliche Veränderungen. Diese Veränderungen sollten politisch ausgetragen werden und nicht durch juristische Schranken unmöglich gemacht werden können. Deshalb kämpfte Abendroth um die wirtschaftspolitische Neutralität des Grundgesetzes und den Begriff der »sozialen Demokratie «, für demokratische und soziale Teilhabe, für Meinungsfreiheit, Demonstrationsfreiheit, das Streikrecht (mit dem Demonstrationsstreik und dem politischen Streik zum Schutze der Verfassung) und gegen die Versuche, den politischen Aktionsraum der Arbeiterbewegung durch Notstandsbefugnisse, strafrechtliche Bestimmungen, Repressalien und Berufsverbote zu beschneiden. ${ }^{23}$

Der Verfassungskampf war für Abendroth in erster Linie ein Kampf um Grundrechte und Verfahrenssicherungen. Für ihn war es kein Nachteil, sondern eine erhebliche Verbesserung der juristischen und politischen Lage, daß die Menschenund Bürgerrechte der amerikanischen und französischen Revolution im Grundgesetz als positives Recht gesetzt sind. ${ }^{24}$ Staatliche Verrechtlichung - sagte er - kann zwar die "Bewußtseinsentwicklung der unterdrückten Klasse hindern«, aber Verrechtlichung bietet der "unterdrückten Klasse« auch die Chance, Verfassungsregeln "zu ihren Gunsten nutzbar" zu machen. ${ }^{25}$ Die Realisierung von Sozialismus war für Abendroth (wie für Marx) nicht primär eine Frage der Menschenrechte, sondern ein Akt der sozialen Befreiung.

\section{*Sozialistische* Verfassung ein hölzernes Eisen?}

Wir finden bei Wolfgang Abendroth keine Theorie der "sozialistischen " Verfassung. ${ }^{26}$ Von seiner - auf Karl Marx und Rosa Luxemburg gestützten Position aus -

- Kann man mit einer bürgerlichen Verfassung eıne sozialistısche Praxıs legitimieren? «, in: Gerhard Stuby, Disziplinterung der Wissenschaft (Mit einem krituschen Nachwort von Ramer Geulen), Frankfurt am Main, O. J. (1970), S. $193 \mathrm{ff.}$

2I S. dazu die emphatısche Betonung eines set - et* gegenüber dem saut ... aut «, die auch hier gitt, Wolfgang Abendroth, Statement, in: Peter Römer, Der Kampf um das Grundgesetz, a.a. O. (Anm. 5), S. 241 .

22 Karl Marx, „Provisorische Statuten der Internationalen Arbeiter-Assozıation « (1864), un: MEW, Bd. I6, S. 14.

${ }_{23}$ Das wird besonders hervorgehoben durch die von Joachim Perels besorgte Edition Wolfgang Abendroths, Arbeiterklasse, Staat und Verfassung, a. a. O. (Anm. I).

24 Von dieser Position aus ist die Unterscheidung von Wolf-Dieter Narr, $\gg$ Der Staat als Appellationsınstanz? $\propto$ in: Vorgänge, Nr. 77, Heft 5, 198 s, unsbes. S.62 ff., zwischen Bürgerrechten als »staatsgegebene Rechte und *vorstaatlichen Rechten* politisch nicht relevant.

25 Statement in: Peter Römer, Der Kampf um das Grundgesetz, a. a. O. (Anm. 5), S. 188.

26 Vgl. in diesem Zusammenhang die Überlegungen zu einer materialistıschen Verfassungstheone*, Thomas Blanke, Das Dilemma der verfassungspoliaschen Diskussion der Linken in der Bundesrepu- 
war die Verfassung der demokratischen Republik, in der es demokratische und soziale Teilhabe gibt, die Verfassung auch einer sozialistischen Gesellschaft solange diese "Staat " noch braucht. Staat und Verfassung waren für Abendroth auch nach einer sozialen Umwälzung stets »Rechtsformen einer bürgerlichen Gesellschaft, eines bürgerlichen Staates «. Da das Ziel des proletarischen Kampfes für ihn nicht ein "sozialistischer" oder "rätemäßiger" Staat war, sondern eine klassenlose und staatenlose Assoziation, worin die freie Entwicklung eines jeden die Bedingung für die freie Entwicklung aller ist ${ }^{28}$, kam es für Abendroth weder auf eine Theorie der "sozialistischen Verfassung" noch auf das Konstruieren irgendeines Rätemodells an. ${ }^{29}$ Der »Übergang zu einer sozialistischen Gesellschaft« war für Abendroth "auf der Grundlage des bestehenden Rechtssystems (etwa in Westdeutschland durch den Transformator des Bekenntnisses zur rechtsstaatlichen sozialen Demokratie in Art. 20 GG vermittelt) « denkbar. ${ }^{30}$ Für ihn war das ein Akt der "Weiterentwicklung der sozialen Demokratie «. ${ }^{31}$ Er hat auch davon gesprochen, "existierende Rechtsregeln " mittels der "Macht des Klassenkampfes durch weitere Rechtsregeln zu ersetzen, die das Interesse dieser unterdrückten Klasse repräsentieren.$^{32}$

Es ist zu bedauern, daß Abendroth seine Auffassung über den Charakter der Verfassung in einer Übergangsperiode nicht systematisch dargelegt hat. Vermutlich befürchtete er, daß eine solche Darlegung gegen seinen Willen im Rahmen des OstWest-Konfliktes gegen die Staaten des »real existierenden Sozialismus « benutzt wird. Es besteht jedoch kein Zweifel, daß Abendroth die Geltung der demokratischen und sozialen Teilhaberechte auch in einem sozialistischen Gemeinwesen für unerläßlich angesehen hat. ${ }^{33}$ Demokratische und soziale Teithabe bedeutet für Abendroth beispielsweise Meinungsfreiheit und Streikrecht ${ }^{34}$ in den Staaten des Ostblocks und zugleich auch personale Freiheit ${ }^{35}$. Für ihn war es undenkbar, daß

blik火, un: Hubert Rottleutner, Hrsg., Probleme marxistischer Rechtstheorie, a.a. O. (Anm. I), S. 419-483; Blanke hat diese Position aufgegeben, s. DDemokratie und Krise in der neueren kritıschen Rechtstheorie*, in: Prokla, Heft 45, Jg.11, Nr. 4 (1981), S. 14 Iff.

27 W. Abendroth in: "Diskussion über Probleme soztalistıscher Rechtspolitik «, ebd., S. 4 I I.

$28 \mathrm{Karl}$ Marx, Fredrich Engels, ${ }^{2}$ Manifest der Kommunistuschen Parteı (1848) in: MEW, Bd. ${ }_{4},{ }_{4} 82$.

29 S. dazu Karl Korsch, »Revolutionäre Kommune* (1931), in: Karl Korsch, Schriften zur Sozzaliszerung, hrsg. von Erich Gerlach, Frankfurr am Main, 1969, S. 107.

30 Wolfgang Abendroth, Arbetterklasse, Staat und Verfassung, a. a. O. (Anm. r), S. 30; Abendroth fügte hunzu: Ob dieser Übergang »friedlich $*$ oder »im gewaltsamen Kampf zur Verterdigung oder Herstellung der politischen Demokratıe erstritten wırd, ... entscherden praktısch nucht die Sozıalisten, sondern die herrschenden Klassen *.

31 Statement in: Peter Römer, Der Kampf um das Grundgesetz, a. a. O. (Anm. 5), S. 192.

32 Ebd., S. 190.

33 Demokratısche Republik und demokratische und sozıale Teilhabe hatten für Abendroth - wie Perels hervorhebt - keınen "instrumentellen Charakter*, sondern geben min weiterer Perspektuve auch die Grundlage für eine sozıalistusche Gesellschaft* ab, Joachim Perels, "Marxismus in der Restaurationszeıt $\alpha$, a.a. O. (Anm. I), S.23; das unterscheldet die Verfassungstheorıe Abendroths grundsätzlich von Staatsrecht der Staaten des "real exıstierenden Soztalismus«; s. dazu z. B. Staatsrecht bürgerlicher Staaten, hrsg. von Wolfgang Menzel u.a., [Ost-]Berlin, 1980, S. 18 r: Der $•$ Kampf um die Bewahrung und Erweiterung demokratischer Rechte* enthält »zugleach die ersten Schritte zur revolutionären Beseitugung der bürgerlichen Demokratıe«, sst - heißt es an anderer Stelle (S. I92) - »Vorbedingung und Bestandteil des Kampfes für Sozıalismus, ... für Grundrechte ener völlig neuen Qualität . Es wäre lohnenswert, Unterschiede und Parallelen zwischen Abendroths Theorie und diesem Lebrbuch herauszuarbeiten.

34 Wolfgang Abendroth, Antagonistische Gesellschaft und politusche Demokratze, a. a. O. (Anm. 4), S. 66; zur Entwicklung in der UdSSR schreibt Abendroth (1956): $\nsim$ das Streikrecht (ist) noch nicht wieder hergestellt๔; vgl. dazu Joachum Perels, »Meinungsfreiheıt als Element des Sozialismusa in: Frankfurter Hefte, Jg. 34, H. 7, 1979, S. 20 ff. u. ders. »Rechtstypus und gesellschaftliche Aneignung*, Norman Paech u. Gerhard Stuby, Hrsg., Wider die herrschende Mennung،, Festschrift für Wolfgang Abendroth, Frankfurt/M., New York, 1982, S. 192.

$35 \mathrm{Vgl}$. dazu Jürgen Seifert, Der unterschedliche Gebrauch des Begriffes >Bourgeoss bes Marx und die Folgen $\%$, in: Hans-Ernst Böttcher, Hrsg., Recht, Justzz, Krtik, Eestschrift für Richard Schmıd, Baden- 
demokratische und soziale Teilhaberechte uminterpretiert werden zu einem "Instrument, um die sozialistische Einheit von Staat und Bürger bewußt herzustellen ${ }^{j 6}{ }^{6}$ Auch die "Diktatur des Proletariats « war in seinen Augen auf die "Ausnahmesituation des akuten Bürgerkrieges « bezogen und sollte durch "Organe der großen Mehrheit der Bevölkerung unter deren ständiger demokratischer Kontrolle ausgeübt werden «. ${ }^{37}$

\section{Die Gefabren der *Waffenstillstandslage*}

Wolfgang Abendroth hat die gegenwärtige Situation als "Waffenstillstandslage beschrieben..$^{38}$ Er spürte, daß sich dadurch auch der Klassenkampf in der zugespitzten Form veränderte, die er kennengelernt hatte. Er hat die Zersetzung der Arbeiterbewegung wahrgenommen. Um dies zu verhindern, war für ihn die Einbeziehung sowohl der Kommunisten als auch der neuen sozialen Bewegungen in den von ihm für notwendig gehaltenen Kampf geboten. Deshalb kam es für ihn darauf an, daß die »überlebenden Köpfe der alten Arbeiterbewegung " und die skritische Jugend " sozialistisches Denken »wieder lebendig machen « (sic.). ${ }^{39}$ Deshalb hat Abendroth immer neu versucht, politisch-soziale Kräfte zu mobilisieren. Deshalb gab es für ihn eine »Pflicht der Arbeiterbewegung ", eine "Verantwortung der Gewerkschaften" und den "geschichtlichen Auftrag der Arbeiterklasse».

Abendroth konnte so sprechen, weil die sozialwissenschaftliche Analyse ihm zeigte, $\mathrm{da}$ es in der Bundesrepublik und auf Weltebene soziale Klassen gibt. Auch Latenzphasen haben nie sein Urteil verändert, daß es in der Bundesrepublik nach wie vor Klassenkampf gibt und zwar in der Regel Klassenkampf von oben in der Form des Freund-Feind-Verhältnisses. ${ }^{\circ} \mathrm{Er}$ ist davon ausgegangen, daß der Kapitalismus auf die Dauer nicht in der Lage ist, das Entstehen von Krisen zu verhindern und daß in Krisenperioden Klassenkräfte notwendig in Bewegung geraten. Abendroth glaubte, auch eine vernichtete, zum Schweigen gebrachte oder gezähmte Arbeiterbewegung kann als militante politisch-soziale Kraft wieder erstehen.

Abendroths politische Theorie ist ein Kind seiner Zeit. Heute hat sich die Klasse der Industriearbeiter in den hochentwickelten, an der Profitmaximierung orientierten Gesellschaften in ihrer Struktur verändert. Auch sie hat Anteil am Sonderstatus der hochentwickelten Industrieländer gegenüber den Massen der unterentwickelt gehaltenen Ländern. Das nationalstaatliche Kampffeld besteht nach wie vor, doch wird es im zunehmenden Maß übelagert durch supra-nationale Ebenen mit einem Übergewicht der Exekutive. Die politıschen Großorganisationen stehen in der Gefahr, von

Baden, 198 5, S. ${ }_{460}$ : Deshalb berühren die Versuche, die Rechte des Privatmenschen zu eliminieren, auch die Rechte des Staatsbürgers.*

36 So Instutut für Theone des Staates und des Rechts der Akademie der Wissenschafien der DDR, Hrsg., Marxistisch-leninistische Staats- und Rechtstheorse, 2. Aufl., Berlin 1975, S. 260.

37 Wolfgang Abendroth, Arbetterklasse, Statat und Verfassung, a. a. O. (Anm. 1), S. 30; hier kann ich Abendroth nicht folgen, obwohl ich seine Gegenposition gegen die marxistisch-leninistusche Doktrin nıcht verkenne; es ist zu unterscheiden zwischen dem sozıologischen und dem staatsrechtlichen Diktaturbegriff; der Begriff .Diktatur des Proletarıats * bedeutet bes Marx, Aufrechterhaltung von Klassenherrschaft; Maßnahmen des Ausnahmezustandes (als staatsrechtliche Diktatur) sollte man als solche bezeıchnen; s. dazu Jürgen Seifert, $\bowtie$ Karl Marx und die Freiheitsrechte $\star$, in: Ossıp K. Flechtheim, Hrsg., Marx heute. Pro und contra, Hamburg, 1983, S. $212 \mathrm{ff}$.

38 Statement, in: Peter Römer, Der Kampf um das Grundgesetz, a. a. O. (Anm. 5), S. 190.

39 Wolfgang Abendroth, Antagonistzsche Gesellschaft und politzsche Demokratue, a. a. O. (Anm.4), S. 462.

$40 \mathrm{~S}$. dazu Jürgen Seifert, "Sozıale Kamptfront oder Freund-Feınd-Lınıe?», in: Sozıalismus, a. a.O. (Anm. I), S. 49 f. 
einem Instrument der sozialen Befreiung zu einer Fessel des Menschen und seiner Befreiung zu werden. Neue Formen des Kampfes und Konfliktlinien unabhängig von den Großorganisationen sind entstanden. Teile des Staatsapparates im Bereich der »Inneren Sicherheit « entziehen sich weitgehend demokratischer Kontrolle und werden angesichts neuer Techniken zu einem Risiko für die demokratische Ordnung. Die Gesetzmäßigkeit der Verwaltung, ein Kernstück einer rechtsstaatlichen und demokratischen Ordnung, gilt nicht mehr in jedem Fall. Die Grenzen setzende Funktion von Verfassung und Gesetz wird durch Generalklauseln und „Funktionserfordernisse" angetastet.

Das alles hat dazu beigetragen, daß sich die politischen und gesellschaftlichen Voraussetzungen der Abendrothschen Theorie verändert haben. Es ist in Zukunft zu prüfen, ob und wenn $j a$, in welchem Umfang das die Abendrothsche Verfassungstheorie berührt. ${ }^{4}$ Ohne Zweifel aber wird diese Theorie dann preisgegeben, wenn ihr Bezugspunkt nicht mehr eine praktisch-tätige politische-soziale Bewegung ist. Dann begegnen wir einer Verselbständigung von Theoriestücken oder der Rückentwicklung der Theorie: Der Kampf um Verfassungspositionen verselbständigt sich, d.h. wird nicht mehr geführt im Blick auf soziale Veränderung, sondern bleibt in citoyenhafter Beschränkung bezogen auf die Ebene des bloß Politischen. Die von Abendroth entwickelten Verfassungsbegriffe, die es ermöglichen, in der Bundesrepublik sozialistische Vorstellungen zu vertreten, werden uminterpretiert zu einklagbaren Rechtsansprüchen auf Reformen. Jede Fundamentalopposition, die den von Abendroth herausgearbeiteten Unterschied zwischen Staatsapparat einerseits und demokratischer Ordnung und Verfassung andererseits aufgibr, kehrt zurück zu der politischen Position, gegen die Abendroth seine Verfassungstheorie entwickelt hat: zur »ultralinken « abstrakten Staatsnegation. ${ }^{+2}$

4t Die Dialektik ist in den Staatsapparat selbst eingegangen. Deshalb kommt es darauf an, ganz im Sinne Abendroths zwischen den einzelnen Teilen des Staatsapparats und den spezifischen Funkuonen zu unterscheiden. Die Datenschutzbeauftragten sind Teil der Exekutuve und stehen partiell im Gegensatz zu Interessen der $*$ Sicherheitsapparate $\alpha$.

42 S. dazu Jürgen Seifert, ’Jenseits von Staatsfixuerung und Staatsnegationa, in: Vorgänge, Nr. 77, Heft 5, 198, S. 37 f. 